\title{
ÜBERLAGERUNGEN DER PROJEKTIVEN EBENE UND HILBERTSCHE MODULFLÄCHEN
}

\author{
Autor(en): Hirzebruch, F. \\ Objekttyp: Article
}

Zeitschrift: L'Enseignement Mathématique

Band(Jahr): 24(1978)

Heft 1-2: L'ENSEIGNEMENT MATHÉMATIQUE

Erstellt am: Mar 8, 2013

Persistenter Link: http://dx.doi.org/10.5169/seals-49691

\begin{abstract}
Nutzungsbedingungen
Mit dem Zugriff auf den vorliegenden Inhalt gelten die Nutzungsbedingungen als akzeptiert. Die angebotenen Dokumente stehen für nicht-kommerzielle Zwecke in Lehre, Forschung und für die private Nutzung frei zur Verfügung. Einzelne Dateien oder Ausdrucke aus diesem Angebot können zusammen mit diesen Nutzungsbedingungen und unter deren Einhaltung weitergegeben werden. Die Speicherung von Teilen des elektronischen Angebots auf anderen Servern ist nur mit vorheriger schriftlicher Genehmigung des Konsortiums der Schweizer Hochschulbibliotheken möglich. Die Rechte für diese und andere Nutzungsarten der Inhalte liegen beim Herausgeber bzw. beim Verlag.
\end{abstract}

\section{SEALS}

Ein Dienst des Konsortiums der Schweizer Hochschulbibliotheken c/o ETH-Bibliothek, Rämistrasse 101, 8092 Zürich, Schweiz

retro@seals.ch

http://retro.seals.ch 


\title{
ÜBERLAGERUNGEN DER PROJEKTIVEN EBENE UND HILBERTSCHE MODULFLÄCHEN ${ }^{1}$
}

\author{
by F. HiRZEBRUCH
}

Wir werden in diesem Vortrag einige verzweigte Überlagerungen der projektiven Ebene als Beispiele von regulären algebraischen Flächen angeben, sie in die Enriques-Kodaira Klassifikation (gemäß der KodairaDimension) einordnen und diese Beispiele, wenn möglich, mit Hilbertschen Modulflächen identifizieren. Beweise werden fortgelassen oder nur skizziert.

Für algebraische Flächen allgemein siehe [8], [10]. Zur Klassifikation Hilbertscher Modulflächen vergleiche [3], [4], [5], [1], [6].

$\S 1$. Eine grobe Klassifikation der kompakten komplexen Mannigfaltigkeiten geschieht mit Hilfe der Kodaira-Dimension. Es sei $X$ eine kompakte komplexe Mannigfaltigkeit der Dimension $n$ und $K$ das kanonische Bündel von $X$. Die Dimension des C-Vektorraumes $H^{0}\left(X, \Omega\left(K^{r}\right)\right)$ der holomorphen Schnitte von $K^{r}(r \geqq 1)$ wird mit $P_{r}$ bezeichnet $(r$-tes Plurigeschlecht, $P_{1}=p_{g}$ ist das geometrische Geschlecht). Man hat eine „meromorphe Abbildung" von $X$ in den komplexen projektiven Raum der Dimension $P_{r}-1$ definiert mit Hilfe einer Basis von $H^{0}\left(X, \Omega\left(K^{r}\right)\right)$. Die Kodaira-Dimension $\kappa(X)$ ist das Maximum der Dimensionen der Bilder aller dieser $r$-fach kanonischen Abbildungen. Sie kann die Werte $-1,0, \ldots, n$ annehmen, wobei $\kappa(X)=-1$ genau dann, wenn alle $P_{r}=0$. Die Kodaira-Dimension ist eine bimeromorphe Invariante.

Wir betrachten reguläre algebraische Flächen $X$, wobei regulär bedeutet, daß die erste Bettische Zahl von $X$ verschwindet. Eine solche Fläche $X$ ist rational genau dann, wenn $\kappa(X)=-1$. In den Fällen $\kappa(X) \geqq 0$ gibt es in der birationalen Äquivalenzklasse von $X$ ein bis auf biholomorphe Äquivalenz eindeutig bestimmtes minimales Modell $X_{0}$, das heißt eine algebraische Fläche, die keine exzeptionellen Kurven (singularitätenfreie rationale Kurven der Selbstschnittzahl -1) enthält. Von $X$ ausgehend kann man $X_{0}$ erreichen, indem man in $X$ alle exzeptionellen Kurven niederbläst (sie sind automatisch disjunkt), in der dann erhaltenen Fläche alle exzep-

1) Vorgetragen im Kolloquium für Topologie und Algebra, Zürich, April 1977. 
tionellen Kurven niederbläst usw. bis man nach endlich vielen Schritten zu $X_{0}$ kommt.

Die erste Chernsche Klasse $c_{1} \in H^{2}(X, \mathbf{Z})$ ist bis auf das Vorzeichen gleich der charakteristischen Klasse von $K$. Die Zahl $c_{1}^{2}[X]$, auch kurz mit $c_{1}^{2}$ bezeichnet, ist eine wichtige Invariante. Die Klassifikation von Enriques-Kodaira (für minimale reguläre Flächen $X$ mit $\kappa(X) \geqq 0$ ) besagt:

$\kappa=0 \Leftrightarrow X$ ist eine $K 3$-Fläche $\left(c_{1}=0\right)$ oder eine Enriques-Fläche $\left(c_{1} \neq 0,2 c_{1}=0\right)$

$\kappa=1 \Leftrightarrow X$ läßt eine eindeutig bestimmte elliptische Faserung über der projektiven Geraden $\mathbf{P}_{1}(\mathbf{C})$ zu. Die Chernsche Klasse $c_{1}$ ist als rationale Kohomologieklasse $\neq 0$ und wird bis auf einen Faktor durch eine Faser repräsentiert. Es ist $c_{1}^{2}=0$

$\kappa=2 \Leftrightarrow c_{1}^{2}>0$

(Flächen mit $\kappa=2$ nennt man vom allgemeinen Typ).

Alle K3-Flächen sind zueinander diffeomorph. Ein Standardbeispiel ist die Fläche $x_{0}^{4}+x_{1}^{4}+x_{2}^{4}+x_{3}^{4}=0$ in $\mathbf{P}_{3}(\mathbf{C})$. Die Fundamentalgruppe einer Enriques-Fläche hat die Ordnung 2. Die universelle Überlagerung ist eine $K 3$-Fläche.

$\S 2$. Wir betrachten die folgende Konfiguration in der reellen projektiven Ebene, bei deren Deutung mir I. Naruki geholfen hat.

Man wähle 4 Geraden in allgemeiner Lage und bezeichne die 6 Schnittpunkte mit $p_{1}, \ldots, p_{6}$. Verbindet man jeden Punkt $p_{i}$ mit demjenigen Punkt $p_{j}$, mit dem $p_{i}$ noch nicht verbunden ist, dann erhält man drei Geraden (die Diagonalen des vollständigen Vierseits) mit drei Schnittpunkten $q_{1}, q_{2}, q_{3}$. Danach verbindet man jeden Punkt $p_{i}$ mit demjenigen $q_{j}$, mit dem $p_{i}$ noch nicht verbunden ist. Man erhält sechs Geraden, von denen sich zwei in jedem $q_{i}$ und drei in jeweils 4 weiteren Punkten $r_{1}, r_{2}, r_{3}, r_{4}$ schneiden. Es gibt genau eine projektive Transformation der projektiven Ebene, welche eine vorgegebene Vertauschung der 4 Geraden bewirkt. Deshalb ist die Gruppe der projektiven Automorphismen unserer Konfiguration isomorph zur symmetrischen Gruppe $S_{4}$. Die $p_{i}$, die $q_{i}$, die $r_{i}$ bilden jeweils einen Orbit.

Man kann die Konfiguration auch so erhalten. Es sei $W$ ein Würfel im $\mathbf{R}^{3}$ mit dem Ursprung 0 als Mittelpunkt. Die Eckpunkte des Würfels, die Mittelpunkte der Kanten, die Mittelpunkte der Seiten verbunden mit 


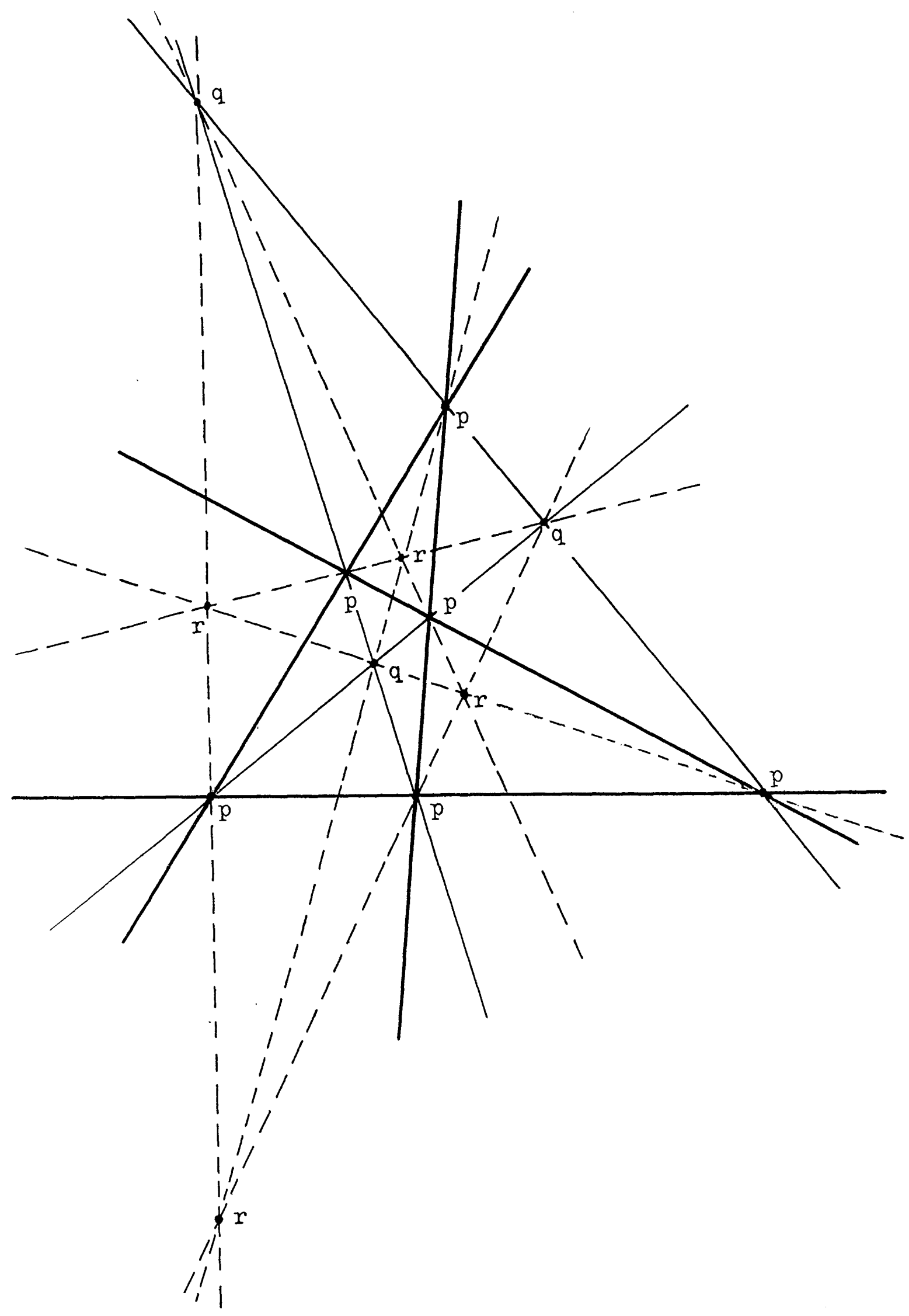

0 definieren 4 bzw. 6 bzw. 3 eindimensionale Teilräume des $\mathbf{R}^{3}$ und damit Punkte von $\mathbf{P}^{2}(\mathbf{R})$. Dies sind die Punkte $r_{i}, p_{i}, q_{i}$; die Gruppe $S_{4}$ wird als Automorphismengruppe von $W$ in SO (3) eingebettet. Es seien $\xi_{1}, \xi_{2}, \xi_{3}$ die Standardkoordinaten des $\mathbf{R}^{3}$. Die quadratische Form $\xi_{1}^{2}+\xi_{2}^{2}+\xi_{3}^{2}$ 
bleibt also bei den Operationen von $S_{4}$ invariant. Wir erweitern die relle projektive Ebene zur komplexen projektiven Ebene $\mathbf{P}_{2}(\mathbf{C})$, homogene Koordinaten $\xi_{1}, \xi_{2}, \xi_{3}$. Der Kegelschnitt $\xi_{1}^{2}+\xi_{2}^{2}+\xi_{3}^{2}=0$ bleibt unter $S_{4}$ invariant. $\mathrm{Er}$ heißt Fundamentalkegelschnitt. $\mathrm{Zu}$ jedem Element $A$ $A \in \mathbf{S O}(3)$ kann man den Kegelschnitt

$$
<\xi,\left(A+A^{t}\right) \xi>=0
$$

einführen. Von besonderem Interesse sind die Kegelschnitte, die zu den Elementen von $S_{4} \subset \mathbf{S O}(3)$ gehören. Wir betrachten die drei Kegelschnitte $E_{1}, E_{2}, E_{3}$, die zu den drei Involutionen der alternierenden Gruppe $A_{4}$ und die vier Kegelschnitte $F_{1}, F_{2}, F_{3}, F_{4}$ die zu den 4 Zyklen der Länge 3 von $A_{4}$ gehören. ( $\mathrm{Zu}$ jedem Zyklus gehören zwei nicht triviale Elemente von $A_{4}$, sie sind invers zueinander und definieren deshalb denselben Kegelschnitt). Die Kegelschnitte $E_{i}, F_{j}$ sind nicht ausgeartet. Rechnerisch läßt sich die Konfiguration der oben angegebenen Geraden zusammen mit den Kegelschnitten besser behandeln, wenn man im $\mathbf{C}^{4}$ (Koordinaten $x_{1}, x_{2}, x_{3}$, $x_{4}$ ) den Unterraum $x_{1}+x_{2}+x_{3}+x_{4}=0$ einführt und dafür die projektive Ebene betrachtet. Die Gruppe $S_{4}$ operiert auf

$$
\mathbf{P}_{2}(\mathbf{C})=\left\{x \in \mathbf{P}_{3}(\mathbf{C}) \mid x_{1}+x_{2}+x_{3}+x_{4}=0\right\}
$$

durch Permutation der homogenen Koordinaten. Es sei $\sigma_{i}$ die $i$-te elementarsymmetrische Funktion der $x_{j}$.

Dann gilt

$$
\begin{array}{ll}
\sigma_{1} \equiv 0 & \\
\sigma_{2}=0 & \text { ist der Fundamentalkegelschnitt. } \\
\sigma_{3}=0 & \text { ist die Vereinigung der } 3 \text { Diagonalen. } \\
\sigma_{4}=0 & \text { ist die Vereinigung der } 4 \text { ursprünglich gegebenen }
\end{array}
$$$$
\sigma_{3}=0 \quad \text { ist die Vereinigung der } 3 \text { Diagonalen. }
$$
Geraden unserer Konfiguration (vollständiges Vierseit).

$\Delta=\prod_{i<j}\left(x_{i}-x_{j}\right)=0$ ist die Vereinigung der im letzten Schritt durch
Verbindung von $p_{i}$ mit $q_{j}$ konstruierten sechs Geraden.

Die Kegelschnitte $E_{1}, E_{2}, E_{3}$ sind gegeben durch

$$
x_{1} x_{2}+x_{3} x_{4}=0, x_{1} x_{3}+x_{2} x_{4}=0, x_{1} x_{4}+x_{2} x_{3}=0 .
$$


Der Kegelschnitt $F_{4}$, der zum Zyklus (123) (4) gehören möge, hat die Gleichung

$$
x_{1} x_{2}+x_{2} x_{3}+x_{3} x_{1}+x_{4}^{2}=0
$$

entsprechend für $F_{1}, F_{2}, F_{3}$.

Die Kegelschnitte $E_{1}, E_{2}, E_{3}$ schneiden sich nur in den Punkten $p_{i}(i=1, \ldots, 6)$ gegeben durch $(0,0,1,-1)$ und Permutationen, und zwar berühren sich in jedem $p_{i}$ genau zwei dieser Kegelschnitte.

Die Kegelschnitte $F_{1}, F_{2}, F_{3}, F_{4}$ schneiden sich nur in den Punkten $q_{i}(i=1,2,3)$ gegeben durch $(1,1,-1,-1)$ und Permutationen, und zwar geht jeder Kegelschnitt $F_{j}$ durch jeden Punkt $q_{i}$, wobei das Schnittverhalten in jedem $q_{i}$ wie folgt ist

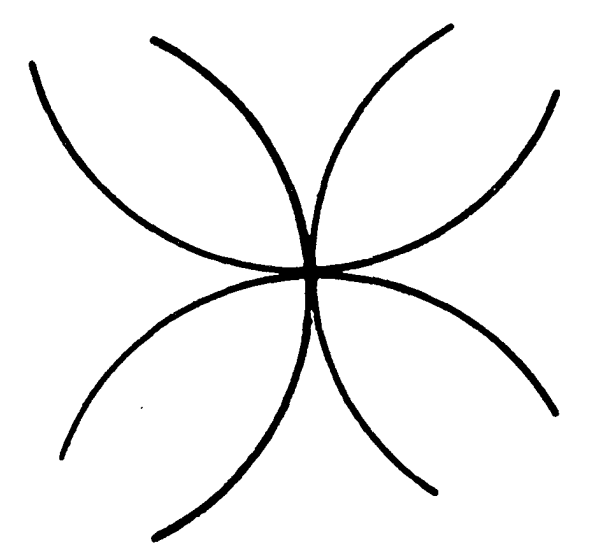

(zwei einfache Berührungen, sonst transversale Schnitte).

Neben dem Fundamentalkegelschnitt gibt es noch zwei Kegelschnitte $B_{1}, B_{2}$, die bei der Gruppe $A_{4}$ in sich übergehen, während sie durch ein Element von $S_{4}$ ungeradem Signums vertauscht werden. (Es sei $\rho=$ $\exp (2 \pi i / 3))$.

$$
\begin{aligned}
B_{1}: & \left(x_{1}+x_{2}\right)\left(x_{3}+x_{4}\right)+\rho\left(x_{1}+x_{3}\right)\left(x_{2}+x_{4}\right) \\
& +\rho^{2}\left(x_{1}+x_{4}\right)\left(x_{2}+x_{3}\right)=0 \\
B_{2}: & \left(x_{1}+x_{2}\right)\left(x_{3}+x_{4}\right)+\rho^{2}\left(x_{1}+x_{3}\right)\left(x_{2}+x_{4}\right) \\
& +\rho\left(x_{1}+x_{4}\right)\left(x_{2}+x_{3}\right)=0
\end{aligned}
$$

Diese quadratischen Ausdrücke kommen als Lagrangesche Resolventen in der Theorie der Gleichung 4. Grades vor. Sie sind Semi-Invarianten für $A_{4}$ bezüglich der beiden nicht-trivialen Charaktere von $A_{4}$, die wegen des Isomorphismus $A_{4} / V_{4} \cong \mathbf{Z} / 3 \mathbf{Z}$ existieren, wobei $V_{4}$ die Kleinsche Vierergruppe ist. Die Kegelschnitte $B_{1}, B_{2}$ gehen durch die 4 Punkte $r_{1}, \ldots, r_{4}$ gegeben durch $(1,1,1,-3)$ und Permutationen. Sie bilden eine Basis des Büschels der durch $r_{1}, r_{2}, r_{3}, r_{4}$ gehenden Kegelschnitte. Schließ- 
lich werden wir noch eine irreduzible Kurve der Ordnung 6 benutzen, nämlich die Kurve

$$
C: 8 \sigma_{2}^{3}+27 \sigma_{3}^{2}=0
$$

Diese Kurve hat in den 4 Punkten $r_{i}$ einen gewöhnlichen Doppelpunkt und in den 6 Schnittpunkten von $\sigma_{3}=0$ mit dem Fundamentalkegelschnitt $\sigma_{2}=0$ eine Spitze. Nach der Plückerschen Formel hat $C$ das Geschlecht 0.

$\S 3$. $\mathrm{Zu}$ jeder Kurve $L$ geraden Grades in $\mathbf{P}_{2}(\mathbf{C})$ gibt es eine wohldefinierte doppelte Überlagerung $X(L)$ von $\mathbf{P}_{2}(\mathbf{C})$ mit $L$ als Verzweigungskurve. Die Fläche $X(L)$ hat singuläre Punkte über den singulären Punkten von $L$. Löst man die Singularitäten von $X(L)$ in der kanonischen minimalen Weise auf, dann erhält man eine singularitätenfreie algebraische Fläche $Y(L)$, die wir in Beispielen untersuchen wollen. Es kommt vor, daß $Y(L)$ nicht regulär ist (vgl. [10] Chap. VIII); in allen unseren Beispielen kann man nachweisen, daß $Y(L)$ regulär ist.

Bsp. 1. Die Verzweigungskurve $L$ sei $\Delta=0$. Dies ist eine Kurve 6. Ordnung (zerfallend in 6 Geraden) mit $q_{1}, q_{2}, q_{3}$ als Doppelpunkten, $r_{1}, \ldots, r_{4}$ als Tripelpunkten und keinen weiteren Singularitäten. Entsprechend hat $X(L)$ sieben singuläre Punkte $\tilde{q}_{i}, \tilde{r}_{j}$. Die Punkte $\tilde{q}_{i}$ werden bei der Auflösung aufgeblasen in eine singularitätenfreie rationale Kurve der Selbstschnittzahl -2 , während $\tilde{r}_{j}$ aufgeblasen wird zu einer Konfiguration singularitätenfreier rationaler Kurven mit folgendem Schnittverhalten

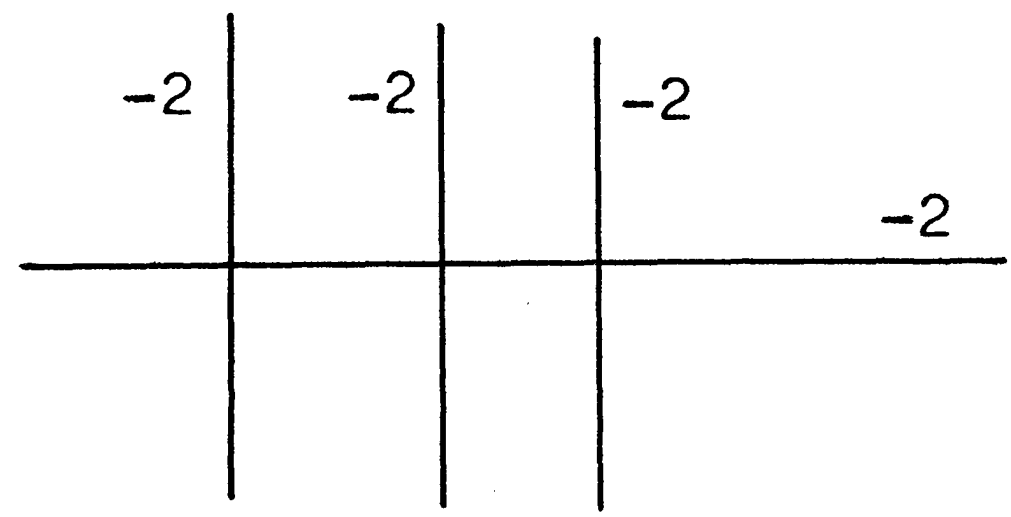

Die Fläche $Y(L)$ ist eine $K 3$-Fläche. Durch die Auflösung der singulären Punkte $\tilde{q}_{i}, \tilde{r}_{j}$ hat man $3+4 \cdot 4=19$ algebraische Zyklen auf $Y(L)$, die als rationale Homologieklassen linear-unabhängig sind bei negativdefiniter Schnittform. Da jede algebraische Fläche einen „Hyperebenenschnitt“ mit positiver Selbstschnittzahl besitzt, ist die Picardsche Zahl 
(Maximalzahl algebraischer Zyklen, die als rationale Homologieklassen linear unabhängig sind) mindestens 20 und deshalb genau gleich 20 , weil für eine $K 3$-Fläche $h^{1,1}=20$ ist. Für $K 3$-Flächen mit maximaler Picardzahl siehe [9].

Bsp. 2. Die Verzweigungskurve $L$ bestehe aus den 4 Geraden $\sigma_{4}=0$ und den drei Kegelschnitten $E_{1}, E_{2}, E_{3}$. Dies ist eine Kurve $L$ der Ordnung 10 mit genau 6 singulären Punkten, nämlich den Punkten $p_{1}, \ldots, p_{6}$. In jedem $p_{i}$ sieht die Verzweigungskurve so aus

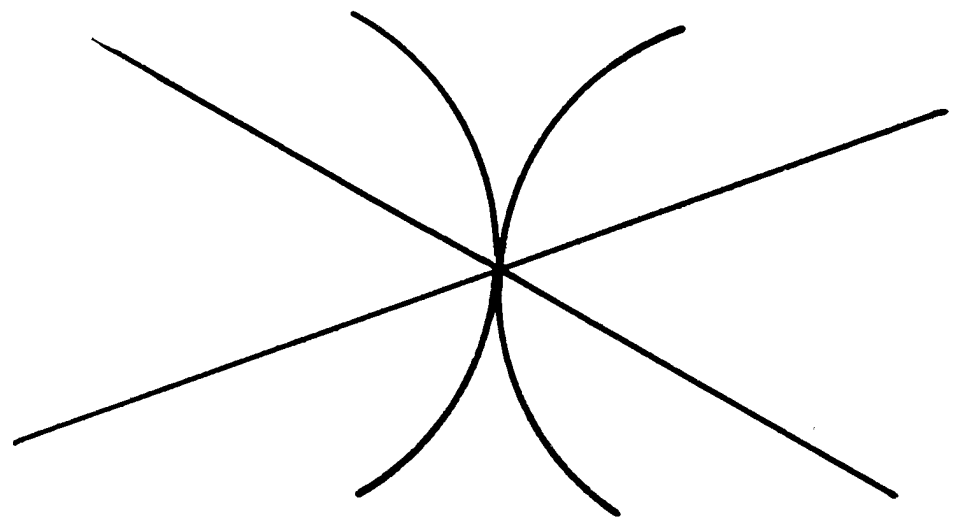

Entsprechend gibt es in $X(L)$ sechs singuläre Punkte $\tilde{p}_{1}, \ldots, \tilde{p}_{6}$. Die Auflösung jedes dieser singulären Punkte wird durch ein Diagram

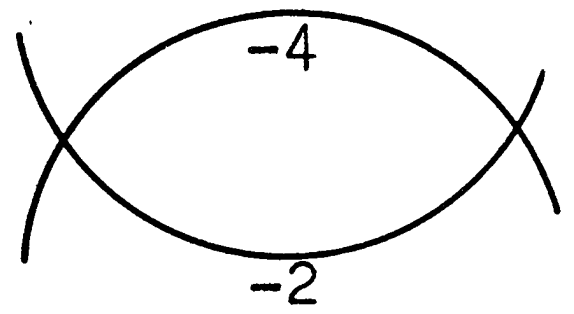

dargestellt (zwei singularitätenfreie rationale Kurven mit Selbstschnittzahl $-4,-2)$. Solche zyklischen Konfigurationen kommen als Auflösungen der Spitzen Hilbertscher Modulflächen vor ([3], vgl. auch [6], wo Singularitäten der Form $Z^{2}=F(X, Y)$ betrachtet werden) und immer, wenn eine zyklische Konfiguration auftritt, kann der Verdacht entstehen, daß es sich um eine Hilbertsche Modulfläche handelt.

Man kann beweisen, daß $Y(L)$ vier disjunkte exzeptionelle Kurven enthält, nämlich die Urbilder der vier Geraden $\sigma_{4}=0$, und $Y(L)$ nach Niederblasen dieser 4 Kurven eine Enriques-Fläche als minimales Modell $Y_{0}(L)$ ergibt.

Bsp. 3. Hier handelt es sich um eine vierblättrige Überlagerung von $\mathbf{P}_{2}(\mathbf{C})$. Wir nehmen zunächst die 2-blättrige Überlagerung entlang des 
Fundamentalkegelschnitts. Das Ergebnis ist isomorph zu $\mathbf{P}_{1}(C) \times \mathbf{P}_{1}(C)$, wobei der Fundamentalkegelschnitt der Diagonale entspricht.

In dieser Fläche betrachten wir als Verzweigungskurve $L$ das Urbild der Vereinigungsmenge der 4 Kegelschnitte $F_{1}, F_{2}, F_{3}, F_{4}$. Da jedes $F_{i}$ den Fundamentalkegelschnitt in 2 Punkten berührt, besteht $L$ aus $8 \mathrm{Kom}$ ponenten (jede einzelne Komponente kann als Graph einer gebrochenen linearen Transformation $\mathbf{P}_{1}(\mathbf{C}) \rightarrow \mathbf{P}_{1}(\mathbf{C})$ angesehen werden). Die Kurve $L$ in $\mathbf{P}_{1}(\mathbf{C}) \times \mathbf{P}_{1}(\mathbf{C})$ hat die folgenden Singularitäten:

a) 6 singuläre Punkte vom Typ (3), die in $\mathbf{P}_{1}(\mathbf{C}) \times \mathbf{P}_{1}$ (C) über $q_{1}, q_{2}, q_{3}$ $\in \mathbf{P}_{2}(\mathbf{C})$ liegen

b) 8 Doppelpunkte, die aus den Berührungspunkten der $F_{i}$ mit dem Fundamentalkegelschnitt entstehen.

Wir bezeichnen die doppelte Überlagerung von $\mathbf{P}_{\mathbf{1}}(\mathbf{C}) \times \mathbf{P}_{\mathbf{1}}(\mathbf{C})$ entlang $L$ mit $X(L)$. Sie hat 14 singuläre Punkte. Die unter b) angegebenen acht Singularitäten von $L$ liefern singuläre Punkte von $X(L)$, die alle in eine singularitätenfreie rationale Kurve der Selbstschnittzahl -2 aufgeblasen werden, während die unter a) angegebenen sechs singulären Punkte von $L$ zu singulären Punkten von $X(L)$ führen, die in eine Konfiguration

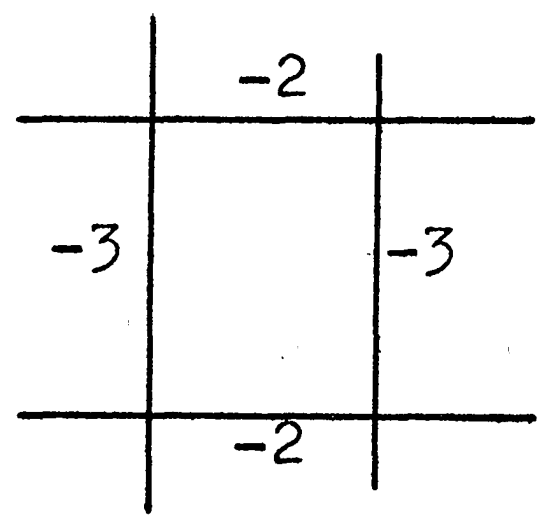

augeblasen werden. Man erhält so eine algebraische Fläche $Y(L)$, von der man nachweisen kann, daß sie regulär ist und Kodaira-Dimension 2 hat (allgemeiner Typ). Sie ist fernerhin minimal. Man kann ausrechnen, daß $Y(L)$ die Eulerzahl 44 und das geometrische Geschlecht $p_{g}=3$ hat. Es folgt $h^{1,1}=36$. Die Picardsche Zahl $\rho$ von $Y(L)$ ist nicht bekannt, doch liefern die Kurven, die von der Auflösung der Singularitäten herrühren, und ein „Hyperebenenschnitt“ die Abschätzung

$$
33 \leqq \rho \leqq 36 \text {. }
$$


Der Vektorraum der homogenen Polynome für die Schar der Kegelschnitte, die durch $q_{1}, q_{2}, q_{3}$ gehen, läßt sich mit $H^{0}(Y(L), \Omega(K))$ identifizieren in Übereinstimmung mit $p_{g}=3$. $\mathrm{Zu}$ diesen Polynomen gehören $F_{1}, F_{2}, F_{3}, F_{4}$. (Beachte, daß die Summe der zugehörigen Polynome (2) gleich 0 ist.) Die einfach-kanonische Abbildung $Y \rightarrow \mathbf{P}_{2}(\mathbf{C})$ ist gleich der Überlagerungsabbildung $Y \rightarrow \mathbf{P}_{2}(\mathbf{C})$ vom Grade 4 gefolgt von einer Cremona-Transformation.

Bsp. 4. Wir betrachten nunmehr als Verzweigungskurve $L$ die Vereinigungsmenge $C \cup B_{1} \cup B_{2}$ (siehe (4) und (5)). Dies ist wieder eine Kurve der Ordnung 10. Man kann leicht ausrechnen, daß $B_{1} \cup B_{2}$ durch die Gleichung $\sigma_{2}^{2}+12 \sigma_{4}=0$ gegeben wird. Also ist $L$ die Kurve

$$
\left(\sigma_{2}^{2}+12 \sigma_{4}\right)\left(8 \sigma_{2}^{3}+27 \sigma_{3}^{2}\right)=0 .
$$

Zunächst müssen die singulären Punkte von $L$ bestimmt werden. Die Kurven $B_{1} \cup B_{2}$ und $C$ schneiden sich nur in $r_{1}, \ldots, r_{4}$ und zwar stimmen die beiden Tangenten von $B_{1} \cup B_{2}$ in $r_{i}$ mit den beiden Tangenten von $C$ in $r_{i}$ überein. Die Kurve $L$ hat also in $r_{i}$ eine Singularität vom Typ (3). Deshalb hat die doppelte Überlagerung $X(L)$ über $r_{i}$ eine Singularität mit der Auflösung

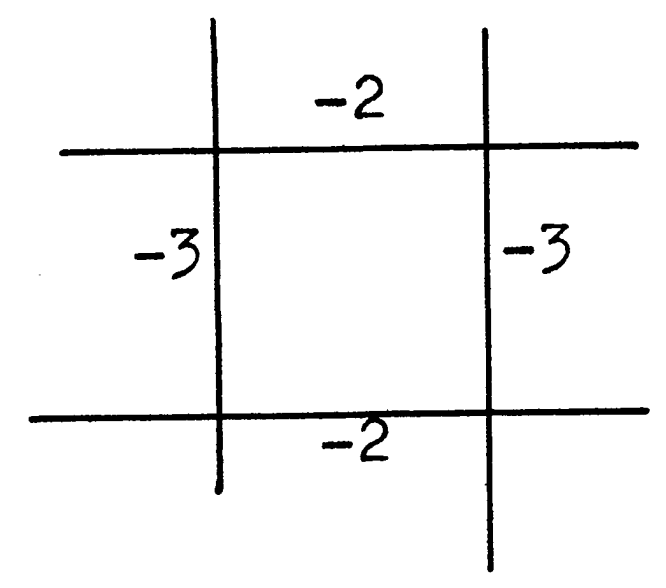

die auch schon in Beispiel 3 vorkam. Ferner hat $C$ und damit $L$ jeweils eine Spitze in den sechs Schnittpunkten von $\sigma_{2}=0$ und $\sigma_{3}=0$. Die entsprechenden 6 Singularitäten von $X(L)$ werden aufgelöst durch je eine Konfiguration

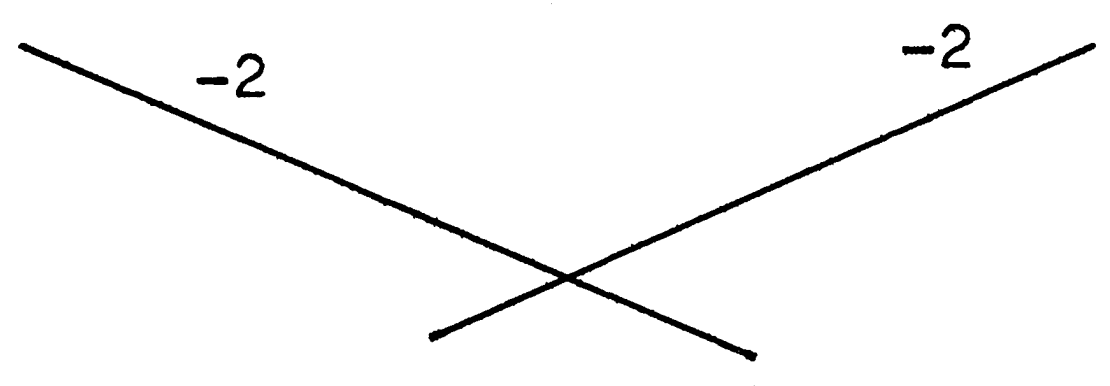


Die so erhaltene algebraische Fläche $Y(L)$ hat Kodaira-Dimension 1, sie ist minimal. Sie hat Eulerzahl 36, das geometrische Geschlecht $p_{g}$ ist gleich 2. Wir werden die elliptische Faserung angeben. Ein von $B_{1}, B_{2}$ verschiedener Kegelschnitt $B$ des durch $B_{1}, B_{2}$ aufgespannten Büschels hat die Schnittzahl 2 mit $C$ in jedem Punkt $r_{i}$. Die Summe der Schnittzahlen von $B$ mit $C$ außerhalb der $r_{i}$ ist deshalb gleich 4 . In $Y(L)$ ist also die Überlagerung eines allgemeinen Kegelschnitts des Büschels eine elliptische Kurve. Damit ist die elliptische Faserung beschrieben. Wir werden noch die Ausnahmefasern angeben. Für die verschiedenen Typen von Ausnahmefasern siehe [7]. Die Kurve $B_{1}$ bildet mit je einer $(-2)$-Kurve aus jeder der 4 Konfigurationen (7) eine Ausnahmefaser vom Typ

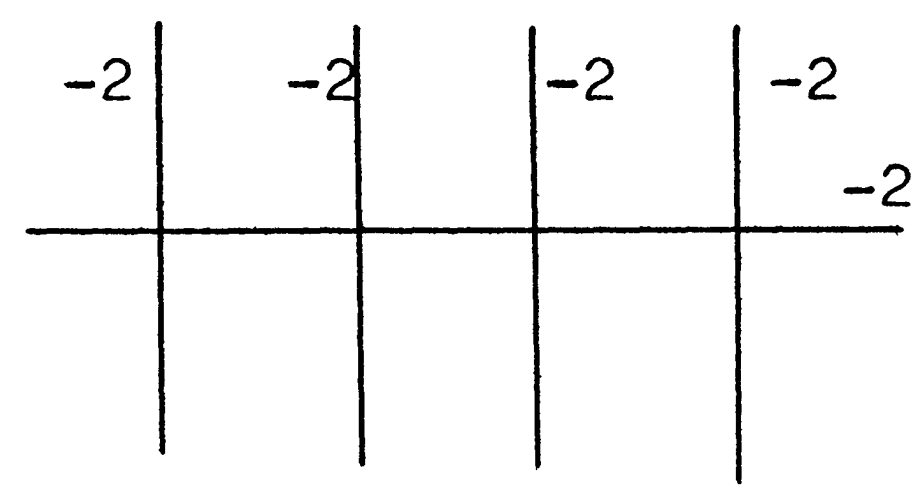

Das entsprechende gilt für $B_{2}$. Die Kurve $\Delta=0$ zerfällt in drei ausgeartete Kegelschnitte des Büschels, jeder bestehend aus zwei Geraden. Jeder dieser ausgearteten Kegelschnitte liefert eine Ausnahmefaser vom Typ

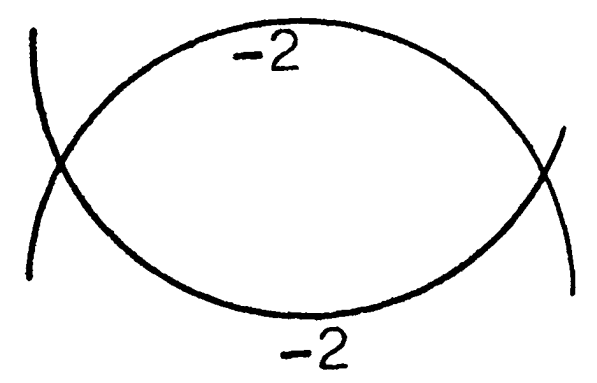

Schließlich müssen noch die Kegelschnitte des Büschels betrachtet werden, welche durch die sechs Schnittpunkte von $\sigma_{2}=0$ und $\sigma_{3}=0$ gehen. Es handelt sich um drei Kegelschnitte. Jeder berührt $\sigma_{2}=0$ in zwei Punkten (nämlich in den Schnittpunkten einer der Komponenten von $\sigma_{3}=0$ mit $\sigma_{2}=0$ ). Die Schnittzahl des Kegelschnitts mit $C$ in diesen beiden Punkten ist jeweils gleich 2, da $C$ in diesen Punkten eine Spitze 
hat. Die 2-blättrige Überlagerung eines solchen Kegelschnittes hat deshalb 2 Komponenten, die zusammen mit zwei der Konfigurationen (8) eine Ausnahmefaser vom Typ

(11)

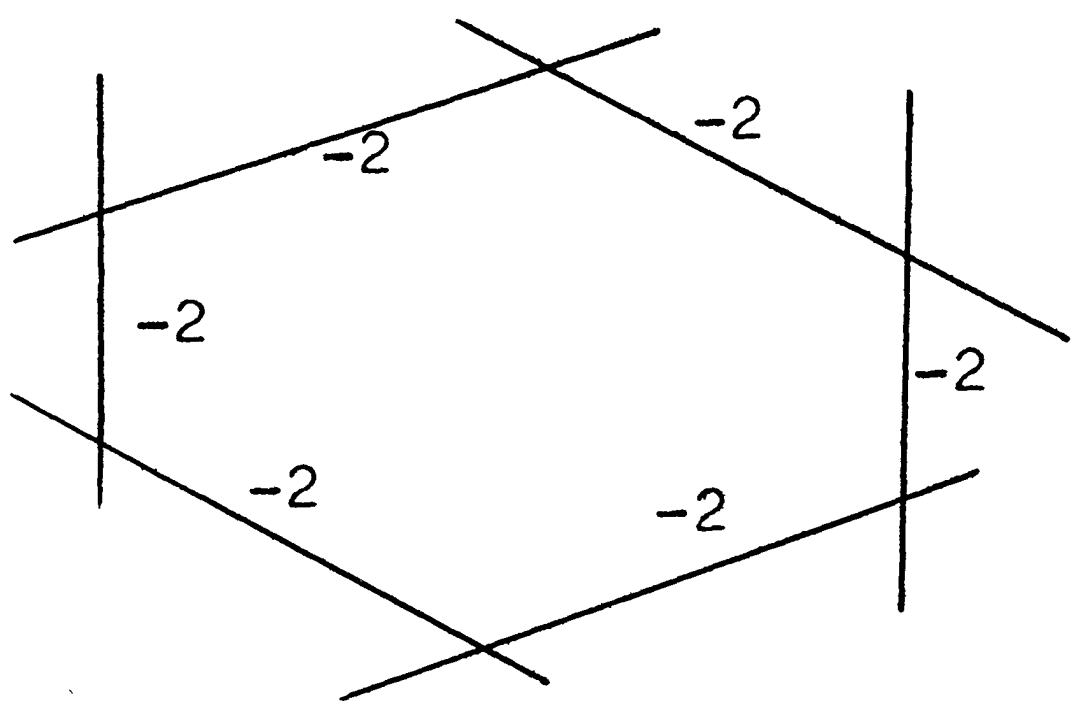

bilden. Es gibt also zwei Ausnahmefasern vom Typ (9), drei vom Typ (10) und drei vom Typ (11). Die Summe der Eulerzahlen der Ausnahmefasern ist in der Tat $2 \cdot 6+3 \cdot 2+3 \cdot 6=36$. Die Picardsche Zahl von $Y(L)$ ist 29 oder $30=h^{1,1}$.

$\S 4$. Es sei $\mathcal{O}$ der Ring der ganzen Zahlen des reell-quadratischen Zahlkörpers $K$. Die Gruppe $G=\mathbf{S L}_{2}(\mathcal{O}) /\{ \pm 1\}$ nennt man die Hilbertsche Modulgruppe des Körpers. Die erweiterte Hilbertsche Modulgruppe $\hat{G}$ wird so erklärt: Es seien $U$ die Gruppe der Einheiten und $U^{+}$die Gruppe der total-positiven Einheiten von $\mathcal{O}$. Es ist

$$
\hat{G}=\left\{\left(\begin{array}{ll}
a & b \\
c & d
\end{array}\right) \mid a, b, c, d \in \mathcal{O}, a d-b c \in U^{+}\right\} /\left\{\left(\begin{array}{ll}
a & o \\
o & a
\end{array}\right) \mid a \in U\right\}
$$

Die Gruppe $\hat{G}$ ist eine Erweiterung von $G$ vom Index 2 , wenn $\mathcal{O}$ keine Einheit negativer Norm besitzt. Sonst ist $\hat{G}=G$. Die Gruppe $\hat{G}$ operiert auf $H^{2}$ und auf $H \times H^{-}$, wo $H$ die obere und $H^{-}$die untere Halbebene von $\mathbf{C}$ ist. Es sei $\tau$ die Involution $\left(z_{1}, z_{2}\right) \mapsto\left(z_{2}, z_{1}\right)$ von $H^{2}$ bzw. $\left(z_{1}, z_{2}\right)$ $\mapsto\left(-z_{2},-z_{1}\right)$ von $H \times H^{-}$. Die auf $H \times H$ bzw. $H \times H^{-}$operierende Gruppe $\hat{G} \cup \hat{G} \tau$ wird mit $\hat{G}_{\tau}$ (symmetrische Hilbertsche Modulgruppe) bezeichnet. Wenn $\Gamma$ eine Untergruppe von $\hat{G}$ von endlichem Index ist, dann sind $H^{2} / \Gamma$ und $\left(H \times H^{-}\right) / \Gamma$ wohldefiniert. Sie lassen sich durch endlich viele Punkte kompaktifizieren (Hinzufügung der Spitzen).

Auf den so erhaltenen kompakten Flächen $\overline{H^{2} / \Gamma}$ und $\overline{\left(H \times H^{-}\right) / \Gamma}$ operiert die Involution $\tau$, wenn im Falle $H^{2}$ der nicht triviale Automor- 
phismus $x \mapsto x^{\prime}$ des Körpers $K$ die Gruppe $\Gamma$ in sich überführt und wenn im Falle $H \times H^{-}$für $A \in \Gamma$ auch $\pm\left(\begin{array}{rr}-1 & 0 \\ 0 & 1\end{array}\right) A^{\prime}\left(\begin{array}{rr}-1 & 0 \\ 0 & 1\end{array}\right)$ zu $\Gamma$ gehört.

Die Quotientenräume $\overline{\left(H^{2} / \Gamma\right)} / \tau$ und $\overline{\left(\left(H \times H^{-}\right) / \Gamma\right)} / \tau$ lassen sich auch als Kompaktifizierung von $H^{2} / \Gamma_{\tau}$ bzw. $\left(H \times H^{-}\right) / \Gamma_{\tau}$ interpretieren, wo $\Gamma_{\tau}=\Gamma \cup \Gamma \tau$ ist. Diese Quotienten nach $\tau$ werden mit $\overline{H^{2} / \Gamma_{\tau}}$ bzw. $\overline{\left(H \times H^{-}\right) / \Gamma_{\tau}}$ bezeichnet.

Die Fläche $\overline{H^{2} / \Gamma}$ hat Singularitäten, nämlich die hinzugefügten Spitzen und Quotientensingularitäten, das sind Punkte von $H^{2} / \Gamma$, die von Punkten in $H^{2}$ herrühren, wo $\Gamma$ eine nicht triviale Isotropiegruppe hat. Löst man alle Singularitäten in kanonischer minimaler Weise auf, dann erhält man eine singularitätenfreie algebraische Fläche $Y_{\Gamma}$, die Hilbertsche Modulfläche zu $\Gamma$. Entsprechendes gilt für die Aktion of $H \times H^{-}$. Wir gelangen so zu einer Fläche $Y_{\Gamma}^{-}$.

Die in $\S 2$ angegebenen Beispiele sind Hilbertsche Modulfächen $Y_{\Gamma}$ oder $Y_{\Gamma}^{-}$, abgesehen von Beispiel 1. Für die Beispiele 2, 3, 4 werden wir jetzt die Gruppe $\Gamma$ angeben. Die Identifizierung von $Y_{\Gamma}^{r}$ oder $Y_{\Gamma}^{-}$mit $Y(L)$ geschieht, indem man in $Y_{\Gamma}$ bzw. $Y_{\Gamma}^{-}$Kurven findet (z.B. gegeben durch die Diagonale $z_{1}=z_{2}$ von $H^{2}$ bzw. $z_{1}=-z_{2}$ von $H \times H^{-}$), die Geraden oder Kegelschnitten unserer Konfiguration entsprechen. Einzelheiten zu Bsp. 2 findet man in [6].

Bsp. 2. Der Körper $K$ ist $\mathbf{Q}(\sqrt{ } 2)$. In $\mathbf{S L}_{2}(\mathcal{O})$ betrachte man die Hauptkongruenz-Untergruppe $\tilde{\Gamma}$ (2) zum Ideal (2). Dann ist $\tilde{\Gamma}(2) /\{ \pm 1\}$ ein Normalteiler $\Gamma(2)$ vom $G=\mathbf{S L}_{2}(\mathcal{O}) /\{ \pm 1\}$ vom Index 48. Die Gruppe $\Gamma$ sei definiert als die Untergruppe von $G$, die von $\Gamma$ (2) und dem Element $\gamma \in G$ erzeugt wird, das durch die Matrix

repräsentiert wird:

$$
\left(\begin{array}{cc}
1+\sqrt{2} & 0 \\
0 & -1+\sqrt{2}
\end{array}\right)
$$

$$
\Gamma=\Gamma(2) \cup \Gamma(2) \gamma
$$

Die Gruppe $\Gamma$ ist eine Erweiterung von $\Gamma$ (2) vom Index 2. Sie operiert frei auf $H^{2}$.

Die Fläche $X(L)$ kann mit $\overline{H^{2} / \Gamma}$ identifiziert werden. Die Spitzen von $\overline{H^{2} / \Gamma}$ entsprechen den sechs singulären Punkten von $X(L)$. Die Fläche $Y(L)$ ist isomorph zu $Y_{\Gamma}$.

Die Gruppe $G / \Gamma$ ist isomorph zu $S_{4}$. Sie operiert auf $\overline{H^{2} / \Gamma}$ und auf $Y_{\Gamma}$. Die durch die Involution $\left(z_{1}, z_{2}\right) \mapsto\left(z_{2}, z_{1}\right)$ von $H^{2}$ auf $\overline{H^{2} / \Gamma}$ und auf 
$Y_{\Gamma}$ induzierte Involution $\tau$ ist mit jedem Element von $G / \Gamma$ vertauschbar. Sie entspricht der Decktransformation von $X(L)$. Man erhält so die Operation von $G / \Gamma \cong S_{4}$ auf $\overline{H^{2} /} \bar{\Gamma}_{\tau} \cong \mathbf{P}_{2}(\mathbf{C})$, von der wir ausgegangen waren. Außerdem folgt, daß der komplexe Raum $\overline{H^{2} / G_{\tau}}$ isomorph ist zum Orbitraum $\mathbf{P}_{2}(\mathbf{C}) / S_{4}$.

Die Hilbertsche Modulfläche $Y_{\Gamma(2)}$ ist eine unverzweigte 2-blättrige Überlagerung von $Y_{\Gamma}$ und zwar ist $Y_{\Gamma(2)}$ eine $K 3$-Fläche mit 8 aufgeblasenen Punkten (vgl. [1]).

Bsp. 3. Der Körper $K$ ist $\mathbf{Q}(\sqrt{3})$. Die Gruppe $G$ hat den Normalteiler $\Gamma$ (2), womit wir auch in diesem Fall die Hauptkongruenz-Untergruppe von $\mathbf{S L}_{2}(\mathcal{O})$ zum Ideal (2) modulo $\{ \pm 1\}$ bezeichnen. Wie erweitern $\Gamma(2)$ mit Hilfe des durch die Matrix

$$
\left(\begin{array}{cc}
2+\sqrt{3} & 0 \\
0 & 2-\sqrt{3}
\end{array}\right)
$$

gegebenen Elements $\gamma \in G$ und definieren wieder

$$
\Gamma=\Gamma(2) \cup \Gamma(2) \gamma
$$

Die Fläche $X(L)$ kann mit $\overline{\left(H \times H^{-}\right) / \Gamma}$ identifiziert werden. Dabei entsprechen die 6 singulären Punkte von $X(L)$, die durch eine Konfiguration

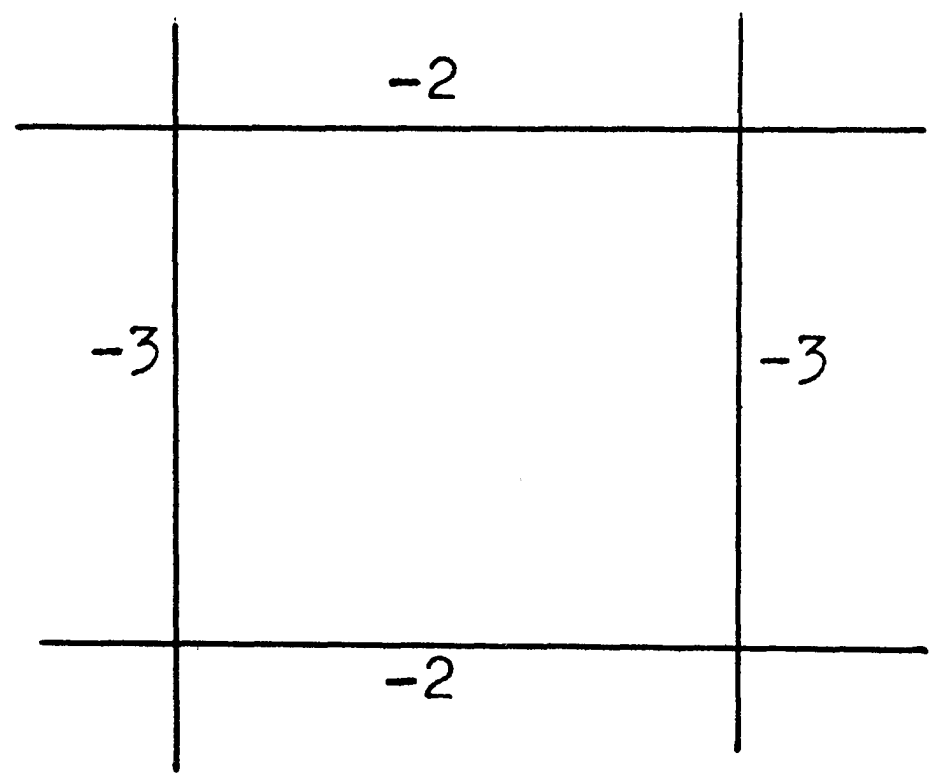

aufgelöst wurden, den Spitzen von $\overline{\left(H \times H^{-}\right) / \Gamma}$, während die übrigen acht Singularitäten den acht $\Gamma$-Äquivalenzklassen in $\mathrm{H} \times \mathrm{H}^{-}$von Punkten mit Isotropiegruppe der Ordnung 2 zugeordnet sind. Die Fläche $Y(L)$ ist isomorph zu $Y_{\Gamma}^{-}$. Wie in Beispiel 2 können wir die Involution $\tau$ auf $\overline{\left(H \times H^{-}\right) / \Gamma}$ und auf $Y_{\Gamma}$ einführen, die wieder mit jedem Element von 
$G / \Gamma$ vertauschbar ist und der Decktransformation von $X(L)$ entspricht. Es gilt

$$
\overline{\left(H \times H^{-}\right) / \Gamma_{\tau}} \cong \mathbf{P}_{1}(\mathbf{C}) \times \mathbf{P}_{1}(\mathbf{C})
$$

wobei $G / \Gamma$ isomorph ist $\mathrm{zu} S_{4}$, und (12) eine äquivariante Isomorphie ist. Die Operation von $S_{4}$ auf $\mathbf{P}_{1}(\mathbf{C}) \times \mathbf{P}_{1}(\mathbf{C})$ ist dabei so zu beschreiben: $S_{4}$ operiert auf $\mathbf{P}_{1}(\mathbf{C}) \cong$ Fundamentalkegelschnitt $\subset \mathbf{P}_{2}(\mathbf{C})$ durch Einschränkung der Aktion auf $\mathbf{P}_{2}(\mathbf{C})$ auf den Fundamentalkegelschnitt, und $S_{4}$ operiert auf $\mathbf{P}_{1}(\mathbf{C}) \times \mathbf{P}_{1}(\mathbf{C})$ durch $g(u, v)=(g(u), g(v))$ für $g \in S_{4}$ und $u, v \in \mathbf{P}_{1}(\mathbf{C})$. Es folgt

$$
\overline{\left(H \times H^{-}\right) / G_{\tau}} \cong\left(\mathbf{P}_{1}(\mathbf{C}) \times \mathbf{P}_{1}(\mathbf{C})\right) / S_{4}
$$

Wir führen nun die erweiterte Hilbertsche Modulgruppe $\hat{G}$ ein. Es ist $[\hat{G}: G]=2$. Die Gruppe $\hat{G} / \Gamma$ ist eine Erweiterung von $G / \Gamma$ vom Index 2, in der Tat ist $\hat{G} / \Gamma$ das direkte Produkt von $G / \Gamma$ und einer Gruppe der Ordnung 2, deren nicht triviales Element $\sigma$ durch ein Element der Ordnung 4 von $\hat{G}$ gegeben wird, dessen Quadrat in $\Gamma$ liegt, und das bis auf einen Faktor durch die Matrix

$$
\left(\begin{array}{cc}
1+\sqrt{3} & 2 \\
-2 & 1-\sqrt{3}
\end{array}\right)
$$

repräsentiert wird. Das Element $\sigma$ operiert auf $\overline{\left(H \times H^{-}\right) / \Gamma_{\tau}}$ und bei der Isomorphie (12) geht diese Operation in die Vertauschung der Faktoren von $\mathbf{P}_{1}(\mathbf{C}) \times \mathbf{P}_{1}(\mathbf{C})$ über. Deshalb folgt

$$
\left(H \times H^{-} / \hat{G}_{\tau} \cong \mathbf{P}_{2}(\mathbf{C}) / S_{4}\right.
$$

Bsp. 4. Der Körper $K$ ist $\mathbf{Q}(\sqrt{ } 3)$. Die Gruppe $\Gamma$ ist die Hauptkongruenz-Untergruppe von $\mathbf{S L}_{2}(\mathcal{O})$ zum Ideal $(\sqrt{ } 3)$, die auch als Untergruppe von $G$ angesehen werden kann.

Die Fläche $X(L)$ kann mit $\overline{H^{2} / \Gamma}$ identifiziert werden. Dabei entsprechen die vier Singularitäten von $X(L)$, die in eine Konfiguration (7) aufgelöst wurden, den Spitzen von $\overline{H^{2} / \Gamma}$, während die 6 Singularitäten von $X(L)$, die in eine Konfiguration (8) aufgelöst wurden, den sechs Quotientensingularitäten von $\overline{H^{2} / \Gamma}$ der Ordnung 3 entsprechen. Die Fläche $Y(L)$ ist mit $Y_{\Gamma}$ zu identifizieren, in [1] wurde diese Fläche mit $Y(12, \sqrt{3})$ bezeichnet. Die Gruppe $G / \Gamma$ ist isomorph zu $A_{4}$, während $\hat{G} / \Gamma$ isomorph $\mathrm{zu} S_{4}$ ist. 
Es folgt wieder

$$
\begin{aligned}
& \overline{H^{2} / G_{\tau}} \cong \mathbf{P}_{2}(\mathbf{C}) / A_{4} \\
& \overline{H^{2} / \hat{G}_{\tau}} \cong \mathbf{P}_{2}(\mathbf{C}) / S_{4}
\end{aligned}
$$

Bemerkung: In den Beispielen 2, 3, 4 ist die Verzweigungskurve in $\mathbf{P}_{2}(\mathbf{C})$ von der Ordnung 10, das hat zur Folge, daß homogene Polynome vom Grad $r$ in den $x_{i}(i=1, \ldots, 4)$ (mit $\sigma_{1} \equiv 0$ ) als Modulformen vom Gewicht $r$ für $\Gamma$ aufgefaßt werden können (vgl. [6]). Daraus können insbesondere die graduierten Ringe $M$ der Modulformen für $G_{\tau}$ bzw. $\hat{G}_{\tau}$ in den Beispielen 2, 3, 4 bestimmt werden. Die Ringe $M$ sind so erklärt wie bei Gundlach [2]. Es gilt (vgl. [2], Sätze 1, 3, 2): Wenn $K=\mathbf{Q}(\sqrt{2})$ und $G_{\tau}$ auf $H^{2}$ operiert, dann

$$
M \cong \mathbf{C}\left[\sigma_{2}, \sigma_{4}, \sigma_{3}^{2}, \sigma_{3} \Delta\right]
$$

modulo einer Relation, die sich aus der Darstellung von $\Delta^{2}$ als Polynom in $\sigma_{2}, \sigma_{3}, \sigma_{4}$ ergibt.

Wenn $K=\mathbf{Q}(\sqrt{3})$ und $\hat{G}_{\tau}$ auf $H \times H^{-}$operiert dann

$$
M \cong \mathbf{C}\left[\sqrt{\sigma_{2}}, \sigma_{4}, \sigma_{3}^{2}\right]
$$

Wenn $K=\mathbf{Q}(\sqrt{3})$ und $\hat{G}_{\tau}$ auf $H^{2}$ operiert, dann

$$
M \cong \mathbf{C}\left[\sigma_{2}, \sigma_{3}, \sigma_{4}\right]
$$

$\S 5$. Bei den Überlegungen in $\S 4$ spielten die Symmetriegruppen $A_{4}$ des Tetraeders und $S_{4}$ des Oktaeders eine Rolle. Wichtig war, daß das betrachtete Ideal, bezüglich dessen die Kongruenz-Untergruppe $\Gamma$ gebildet wurde, die Diskriminante teilt. Daraus folgte, daß die Involution $\tau$ mit jedem Element von $\hat{G} / \Gamma$ vertauschbar ist. Diese Bemerkung ist in vielen anderen Fällen nützlich. Hier soll nur noch ein Beispiel erwähnt werden, das in [6] ausführlich besprochen wurde. Es handelt sich um die Symmetriegruppe $A_{5}$ des Ikosaeders. Sie operiert auf $\mathbf{P}_{2}(\mathbf{C})$, die Aktion kommt von einer 3-dimensionalen Darstellung von $A_{5}$. Der Ring der invarianten Polynome (vgl. [6]) wird nach Felix Klein von Polynomen $A, B, C, D$ der Grade 2, 6, 10 bzw. 15 erzeugt. Klein gibt eine ganz bestimmte Kurve $C=0$ an. Sie ist irreduzibel, hat sechs Doppelspitzen und ist deshalb nach der Plückerschen Formel rational. Die 2-blättrige Überlagerung entlang der Kleinschen Kurve $L$ (gegeben durch $C=0$ ) ergibt eine Fläche $X(L)$ mit sechs singulären Punkten. Nach Auflösung der Singularitäten 
ergibt sich eine algebraische Fläche $Y(L)$, die man als rational nachweisen kann.

Es sei nun $K=\mathbf{Q}(\sqrt{5})$ und $\Gamma$ die Hauptkongruenz-Untergruppe von $G=\mathbf{S L}_{2}(\mathcal{O}) /\{ \pm 1\}$ zum Ideal $(\sqrt{5})$. Dann ist $\overline{H^{2} / \Gamma}$ isomorph zu $X(L)$, wobei die sechs singulären Punkte von $X(L)$ den Spitzen von $\overline{H^{2} / \Gamma}$ entsprechen. Ferner ist $Y_{\Gamma}$ mit $Y(L)$ zu identifizieren. Es ist

$$
\begin{aligned}
& {\overline{H^{2} / \Gamma_{\tau}}}_{\bar{H}^{2} / G_{\tau}} \cong \mathbf{P}_{2}(\mathbf{C}) \\
& \mathbf{P}_{2}(\mathbf{C}) / A_{5}
\end{aligned}
$$

und der Ring $M$ der Modulformen zu $G_{\tau}$ ist gegeben durch

$$
M \cong \mathbf{C}[A, B, C, D]
$$

modulo einer von Klein angegebenen Relation, die $D^{2}$ als Polynom in $A, B, C$ ausdrückt.

\section{LITERATUR}

[1] VAN DER GeER, G. B. M. On Hilbert modular surfaces of principal congruence subgroups. Dissertation, Rijksuniversiteit te Leiden, 1977.

[2] Gundlach, K.-B. Die Bestimmung der Funktionen zu einigen Hilbertschen Modulgruppen. Journal f.d.r.u.a. Math. 220 (1965), pp. 109-153.

[3] Hirzebruch, F. Hilbert modular surfaces. L'Enseignement Math. 19 (1973), pp. 183281.

[4] Hirzebruch, F. and A. VAN de Ven. Hilbert modular surfaces and the classification of algebraic surfaces. Invent. Math. 23 (1974), pp. 1-29.

[5] Hirzebruch, F. and D. ZaGier. Classification of Hilbert modular surfaces, in "Complex Analysis and Algebraic Geometry", Iwanami Shoten und Cambridge Univ. Press 1977, pp. 43-77.

[6] Hirzebruch, F. The ring of Hilbert modular forms for real quadratic fields of small discriminant, in Modular Functions of One Variable VI (Bonn 1976), Lecture Notes in Mathematics, vol. 627 (1977), pp. 287-323. Springer-Verlag, BerlinHeidelberg-New York.

[7] Kodaira, K. On compact analytic surfaces II. Ann. of Math. 77 (1963), pp. 563-626.

[8] Š Afarevič, I. R. Algebraic surfaces. Proceedings Steklov Institute Math. 75 (1965); ins Englische übersetzt: Amer. Math. Soc. Providence, Rhode Island, 1967.

[9] Shioda, T. and H. Inose. On singular K3 surfaces, in "Complex Analysis and Algebraic Geometry", Iwanami Shoten und Cambridge Univ. Press 1977, pp. 119136.

[10] Zariski, O. Algebraic surfaces. Ergebnisse der Mathematik Bd. 61, Second supplemented edition, Springer-Verlag, Berlin-Heidelberg-New York, 1971.

F. Hirzebruch

Mathematisches Institut

der Universität Bonn

Bundesrepublik Deutschland 